\title{
Overexpression of Brassica napus MPK4 Enhances Resistance to Sclerotinia sclerotiorum in Oilseed Rape
}

\author{
Zheng Wang, Han Mao, Caihua Dong, Ruiqin Ji, Li Cai, Hao Fu, and Shengyi Liu \\ The Key Laboratory of Genetic Improvement for Oil Crops, the Ministry of Agriculture, P. R. China, and Department \\ of Functional Genomics and Molecular Biology, Oil Crops Research Institute, Chinese Academy of Agricultural Sciences, \\ Wuhan 430062, P. R. China
}

Submitted 7 August 2008. Accepted 8 November 2008.

Sclerotinia sclerotiorum causes a highly destructive disease in oilseed rape (Brassica napus) resulting in significant economic losses. Studies on the Arabidopsis thaliana MPK4 loss-of-function mutant have implicated that AtMPK4 is involved in plant defense regulation, and its effect on disease resistance varies in different plantpathogen interactions. In this study, we isolated a $B$. napus mitogen-activated protein kinase, $B n M P K 4$, and found that $B n M P K 4$ along with $P D F 1.2$ are inducible in resistant line Zhongshuang9 but both are consistently suppressed in susceptible line 84039 after inoculation with $S$. sclerotiorum. Transgenic oilseed rape overexpressing BnMPK4 markedly enhances resistance to $S$. sclerotiorum and Botrytis cinerea. Further experiments showed that transgenic plants inhibited growth of $S$. sclerotiorum and constitutively activated $P D F 1.2$ but decreased $\mathrm{H}_{2} \mathrm{O}_{2}$ production and constitutively suppressed $P R-1$ expression. Treatment of roots of the transgenic plants with $\mathrm{H}_{2} \mathrm{O}_{2}$ solution resulted in enhanced susceptibility to the two pathogens. Our results support the idea that MPK4 positively regulates jasmonic acid-mediated defense response, which might play an important role in resistance to $S$. sclerotiorum in oilseed rape.

Plants respond actively to pathogen attack by deploying a range of multifaceted defense response mechanisms which culminate in resistance. Apart from specific recognition typified by the gene-for-gene relationship, plants also possess the capacity for a nonspecific, wide-ranging enhanced defense activation which operates after inoculation with a pathogen (Hammerschmidt 1999). Studies on host-pathogen interac-

Current address for R. Ji: College of Horticulture, Shenyang Agricultural University, Shenyang 110161, P. R. China.

Current address for L. Cai and H. Fu: Ningbo Institute of Technology, Zhejiang University, Ningbo 315100, Zhejiang, P. R. China.

Current address for L. Cai: College of Plant Science and Technology, Huazhong Agricultural University, Wuhan 430070, China.

Corresponding author: S. Liu; Telephone: +1 86-27-86812896; Fax: +1 8627-86816451; E-mail: liusy@ oilcrops.cn

Brassica napus MPK4 nucleotide sequence data has been deposited in the GenBank database under accession number EU581868.

* The $\boldsymbol{e}$-Xtra logo stands for "electronic extra" and indicates that three supplemental figures are published online. tions in the model plant Arabidopsis thaliana have established that defense responses against microbial pathogens are regulated by a network of interconnecting signaling pathways in which the plant signal molecules salicylic acid (SA), jasmonate (JA), and ethylene (ET) play a dominant role (Dong 1998; Feys and Parker 2000; Glazebrook et al. 2003; Martinez et al. 2001). SA, JA, and ET are also known to regulate the transcript levels of defense genes in many other plant species (Dong 1998). For example, as defense marker genes, $P R-1$ and PDF 1.2 have been transcriptionally investigated in Brassica napus and B. rapa (Liu et al. 2007; Takemoto et al. 2005). In most cases, the SA-mediated signaling pathway provides protection from biotrophic fungi, oomycetes, and bacteria such as Erysiphe orontii, Hyaloperonospora (formerly Peronospora) parasitica, and Pseudomonas syringae, while the JA/ET-mediated signaling pathway activates defense responses against many necrotrophic fungi, such as Alternaria brassicicola and Botrytis cinerea (Glazebrook 2005; Penninckx et al. 1996; Thomma et al. 1998).

Plant mitogen-activated protein kinases (MAPK) have been implicated in plant defense regulation as a new component of defense signaling pathways. MAPK are conserved in eukaryotic signal transduction, where they orchestrate responses to extracellular stresses and developmental cues. MAPK activity is controlled by sequential activation of three protein kinases, by which an MAPK kinase kinase (MAPKKK) activates an MAPK kinase (MAPKK) that in turn activates an MAPK (Madhani et al. 1997). Arabidopsis MAPK 4 null mutant (Atmpk4) constitutively expresses SA-mediated defense responses and exhibits enhanced resistance to the virulent pathogens $P$. syringae and Peronospora parasitica (Petersen et al. 2000) but fails to induce expression of defense marker genes in JA/ET pathways and exhibits increased susceptibility to the necrotrophic pathogen A. brassicicola (Brodersen et al. 2006; Petersen et al. 2000). MEKK1 is essential for activation of MPK4, and expression of $P R-1$ is constitutively upregulated in the mekk1 mutant, whereas that of PDF1.2 is slightly downregulated (Ichimura et al. 2006). Similarly, Arabidopsis EDR1 functions as an MAPKKK to negatively regulate SA-inducible defense responses to E. cichoracearum (Frye et al. 2001). Interestingly, Arabidopsis plants expressing constitutively active MKK2 exhibit activation of MPK4 but enhanced resistance against $P$. syringae and impaired resistance against infection by A. brassicicola (Brader et al. 2007). In tobacco, Nicotiana tabacum MPK4 (NtMPK4) and N. attenuate MPK4 (NaMPK4) are involved in JA signaling and response to ozone as well as herbivore attack (Gomi et al. 2005; Wu et al. 2007). To further 
understand its role in disease defense, it is necessary to examine whether and how MPK4 responds to other pathogens.

The roles of different plant MAPK appear to be complex and diverse when considering disease resistance. In contrast to MPK4, some MAPK play a positive role in regulating disease defense responses associated with hypersensitive response (HR) and systemic acquired resistance (SAR). WIPK and SIPK are activated in the Cf-9/Avr9 resistance-avirulence (R-Avr) interaction and positively regulate resistance in tobacco, and their Arabidopsis orthologs MPK3 and MPK6 function in flagellininduced resistance activation (Asai et al. 2002; Menke et al. 2004; Romeis et al. 1999). The specific receptor FLS2 acts upstream of the MAPKKK AtMEKK1, which utilizes the two highly conserved MAPKK AtMKK4 and AtMKK5 (which are highly similar to NtMEK2) to activate AtMPK3 and AtMPK6 (Asai et al. 2002). The MPK3/MPK6 cascade regulates camalexin synthesis through transcriptional regulation of the biosynthetic genes after pathogen infection (Ren et al. 2008). Arabidopsis MKK7 is induced by pathogen infection. The increased expression of $M K K 7$ results in accumulation of elevated levels of SA, constitutive pathogenesis-related $(P R)$ gene expression, and enhanced resistance to $P$. syringae and $H$. parasitica (Zhang et al. 2007). Reducing mRNA levels of $M K K 7$ by antisense RNA expression not only compromises basal resistance but also blocks the induction of SAR. In tomato, LeMAPKKKa, an MAPKKK, is a positive regulator of cell death associated with both plant immunity and disease development (del Pozo et al. 2004). Epitasis experiments placed LeMAPKKKa upstream of the tomato homologs of NtMEK2 and NtSIPK.

Within the Arabidopsis genome sequence, 60 genes are predicted to encode MAPKKK, 10 genes to encode MAPKK, and 20 genes to encode MAPK (Ichimura et al, 2002). Although there is likely to be some degree of functional redundancy, the high number of genes for MAPK cascade components indicates that plants rely heavily upon MAPK cascades for signal transduction (Pedley and Martin 2005). This is at least indicated by the fact that there is a divergence of positive and negative regulation roles of different MAPK cascades, and both loss (silencing or T-DNA insertion) and gain (overexpression) of functions in each single locus have their corresponding phenotypes (not completely compensated by other loci). To our knowledge, however, there has not been a specific report examining whether overexpression of MPK4 in plants affects disease defense.

Sclerotinia sclerotiorum is a necrotrophic ascomycete (Guo and Stotz 2007). This destructive pathogen attacks more than 400 plant species (Boland and Hall 1994) and has a worldwide distribution. In oilseed rape, one of the most important oil crops in the world, it causes rotting of leaves, stems, and pods, resulting in a considerable seed yield loss in China and other regions of the world. No immune or highly resistant cultivars of oilseed rape have been reported to date, and few genetic sources of resistance to the pathogen are available to breeders (Liu et al. 2005). Control of the disease depends heavily on

A
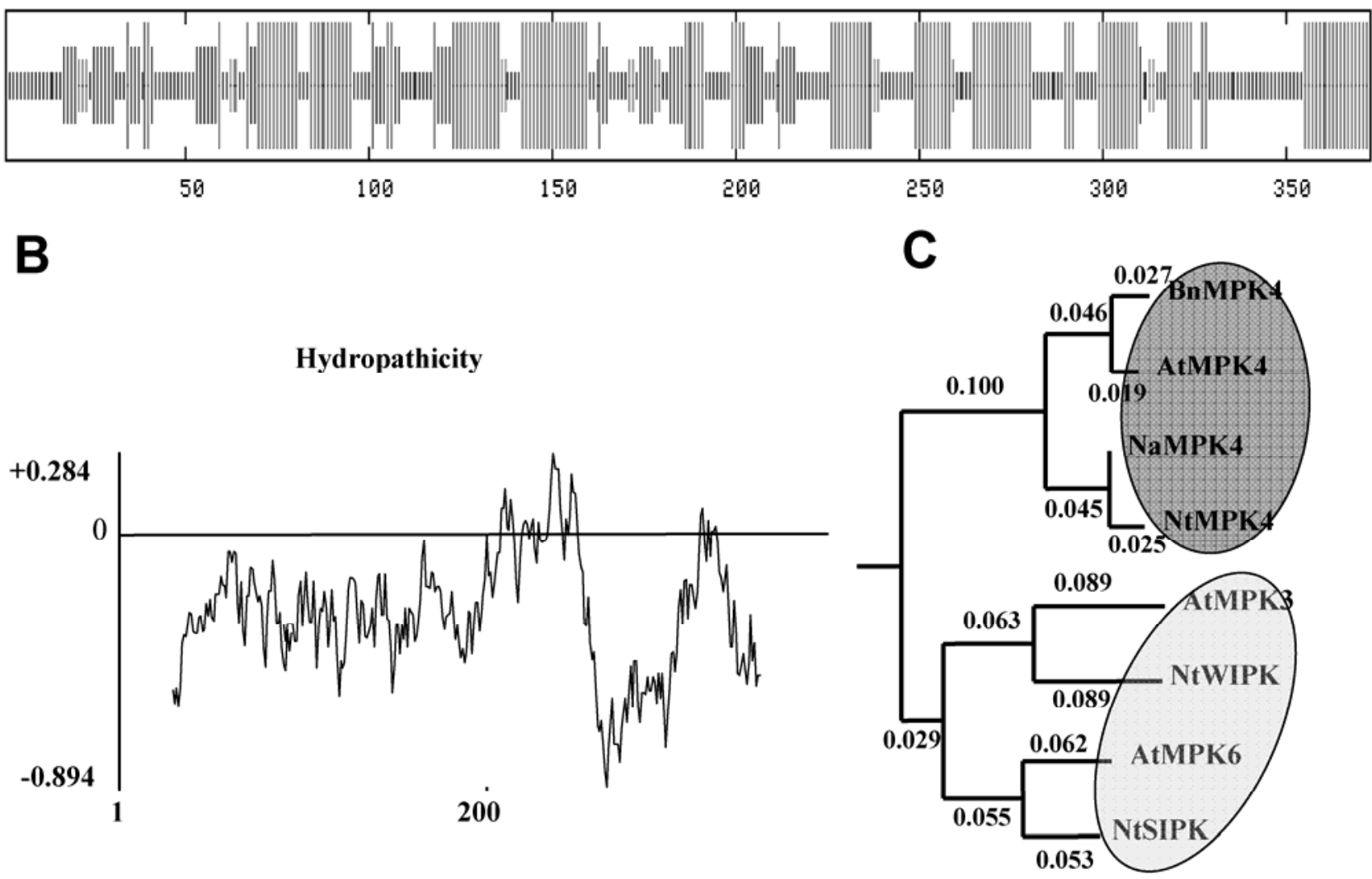

Fig. 1. Characteristic analysis of a BnMPK4 sequence. A, SOPMA analysis of BnMPK4 secondary structure: $\alpha$ helix, extended strand, $\beta$ turn, and random coil are indicated with the longest, second longest, second shortest, and shortest vertical lines, respectively. B, Hydropathicity analysis of BnMPK4 with Vector NTI Suite 10.0. The vertical axis indicates the average hydropathicity and the horizontal axis indicates the individual amino acids. C, Phylogenetic analysis of BnMPK4 and other mitogen-activated protein kinases (MAPKs) involved in plant defense. The tree is constructed with the PHYLIP programs. The branch lengths are proportional to the sequence divergence. Numbers indicate genetic distance. Accession numbers are as follows: Brassica napus MPK4 (BnMPK4, EU581868); Arabidopsis thaliana MPK4 (AtMPK4, NM_116367), MPK3 (AtMPK3, NM_114433), and MPK6 (AtMPK6, NM_129941); Nicotiana tabacum MPK4 (NtMPK4, BAE46985), WIPK (NtWIPK, D61377), and SIPK (NtSIPK, U94192); and N. attenuata MPK4 (NaMPK4, EF121307). 
application of fungicides to the crop, but this is expensive and can be ineffective due to the difficulties associated with applying sprays to thick canopies and a lack of suitable forecasting methods to enable timely application of fungicides. So far, attempts have been made to engineer disease resistance in economically important crop plants. However, the molecular basis of plant defense to this pathogen remains poorly understood, which restricts engineering resistance by transgenic approaches.

Here, we isolated an oilseed rape cDNA clone encoding an MAPK B. napus MPK4 (BnMPK4) and we overexpressed $B n M P K 4$ to investigate its role in defense response to $S$. sclerotiorum in oilseed rape.

\section{RESULTS}

\section{Isolation of $\mathrm{BnMPK4}$}

from oilseed rape and its structure analysis.

A cDNA encoding a homolog of AtMPK4 was cloned from a normalized cDNA library of B. napus cv. Zhongshuang9. The library was constructed from mixed mRNAs of $B$. napus leaves treated with the fungi $S$. sclerotiorum or chemical benzothiadiazole (BTH), methyl jasmonate (MeJA), or oxalic acid (OA, a toxin and pathogenicity factor produced by S. sclerotiorum). The entire open reading frame of the cloned cDNA encodes a protein of 373 amino acid residues that exhibit $95 \%$ identity with AtMPK4 (NM_116367). Similar to AtMPK4, the protein sequence contains a conserved amino acid motif $\left(\mathrm{T}_{201} \mathrm{E}_{202} \mathrm{Y}_{203}\right)$ at the phosphorylation site and a common docking (CD) domain in its $\mathrm{C}$-terminal extension. The $\mathrm{CD}$ domain functions as a docking site for MAPKK, phosphatases, and protein substrates (Ichimura et al. 2002). Thus, the cloned cDNA was designated as BnMPK4 (GenBank accession no. EU581868). The MAPK alignment using the ClustalX programs indicated that BnMPK4 belongs to the B subgroup of the TEY subtype.

SOPMA analysis revealed that the overall folding of BnMPK4 is basically built with $\alpha$-helixes (41.29\%) and random coils $(39.68 \%)$ that are connected with extended strands (13.94\%) and $\beta$-turns (5.09\%) (Fig. 1A). Hydropathicity analysis by using Vector NTI Suite 10.0 showed that BnMPK4 is a hydrophobic protein (Fig. 1B). IPSORT Prediction showed that the value of average negative charge (FAUJ880112) in the 1-30 substring is only 0.0666667 , and that BnMPK4 has a chloroplast transit peptide and a mitochondrial targeting peptide, suggesting that BnMPK4 might transit among cellular organelles.

Analysis of phylogenetic relationships would help provide initial insights into degree of structural and functional conservation. Several plant MAPK, including AtMPK4, AtMPK3, AtMPK6, NtMPK4, NtWIPK, NtSIPK, and NaMPK4, were implicated in plant defense (Asai et al. 2002; Gomi et al. 2005; Grant and Loake 2000; Menke et al. 2004; Romeis et al. 1999; $\mathrm{Wu}$ et al. 2007). Based on alignments (Supplementary Fig. 1) using ClustalX with default parameters, tree topologies were generated by the PHYLIP programs PROTDIST and NEIGHBOR (Felsenstein 1989). Neighbor-joining trees were drawn (Fig. 1C). Results showed that there are two distinct subfamilies of these MAPK and that MPK4s differ from several other MAP kinases, which might indicate a potentially functional difference between them.

\section{Response of BnMPK4}

\section{to $S$. sclerotiorum infection in oilseed rape.}

The Arabidopsis MPK4 loss-of-function mutant exhibits increased susceptibility to the necrotrophic fungus $A$. brassicicola, which is accompanied by decreased expression of PDF1.2 (Brodersen et al. 2006). In order to determine whether
$B n M P K 4$ functions in defense responses to the necrotrophic fungus $S$. sclerotiorum, we detected expression of BnMPK4 in oilseed rape infected by $S$. sclerotiorum using quantitative reverse-transcription polymerase chain reaction (RT-PCR). The results showed that $B n M P K 4$ transcription was significantly $(P<0.05)$ upregulated $(1.70$-fold) in the resistant cv. Zhongshuang9 whereas expression of the gene was significantly $(P<$ $0.05)$ downregulated (0.42-fold) in the susceptible cv. 840396 h after S. sclerotiorum inoculation (Fig. 2), although the transcription in both cultivars was downregulated $24 \mathrm{~h}$ after the inoculation. A similar result was also detected in PDF1.2 expression that was significantly $(P<0.05)$ upregulated $(4.60$ fold) and significantly $(P<0.05)$ downregulated $(0.36$-fold $)$ in the two lines, respectively (Fig. 2). These primary results led us to conduct further studies on BnMPK4.

\section{Overexpression of $B n M P K 4$ in oilseed rape.}

To further functionally characterize $B n M P K 4$, we constructed a plant transformation vector containing the Cauliflower mosaic virus (CaMV) $35 \mathrm{~S}$ enhancer, double CaMV $35 \mathrm{~S}$ promoter, $\Omega$ sequence (improving protein translation), $B n M P K 4$ cDNA, and the CaMV Nos terminator in its T-DNA region and transformed oilseed rape. Cv. 84039, in which BnMPK4 was suppressed after S. sclerotiorum inoculation, was chosen as the recipient for transformation. Five independent transgenic lines overexpressing $B n M P K 4$ were generated and confirmed by PCR using a pair of primers (one specific to CaMV $35 S$ promoter and another specific to the MPK4 cDNA), Southern blot using a probe specific to the $35 \mathrm{~S}$ promoter (to avoid nonspecific hybridization), and Northern blot using a probe specific to BnMPK4 cDNA fragments. As ex-
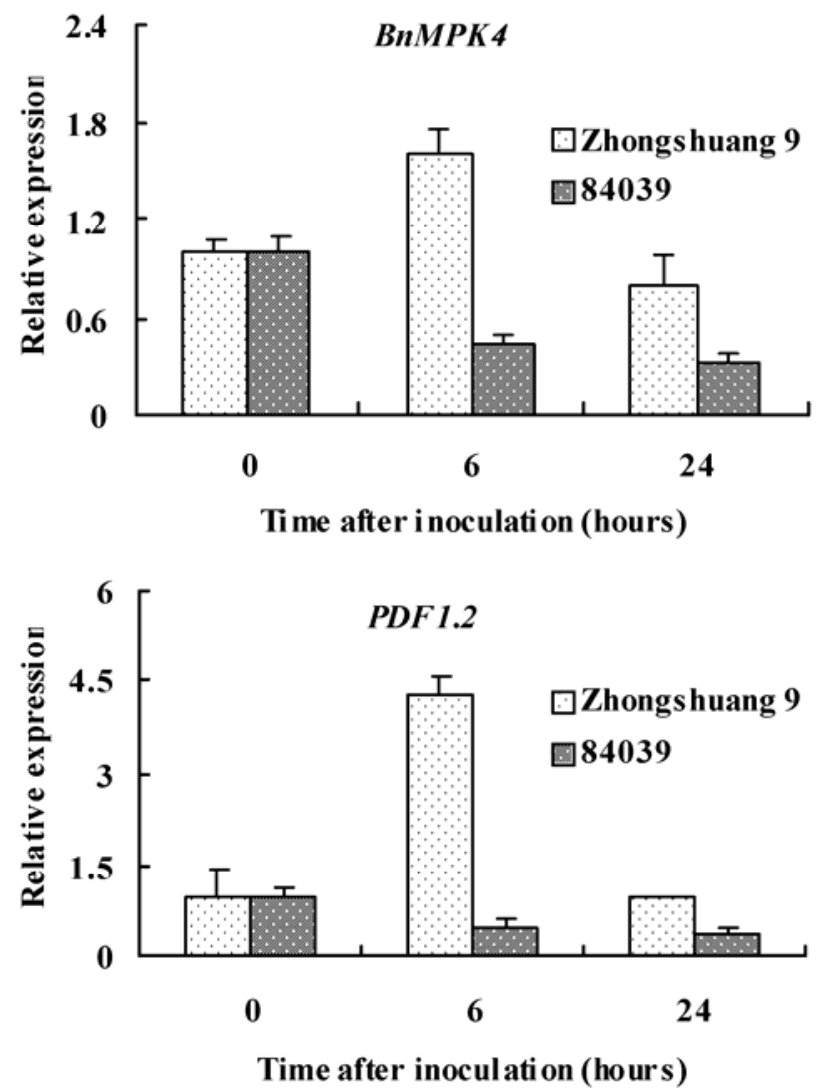

Fig. 2. Responses of BnMPK4 and PDF1.2 to Sclerotinia sclerotiorum. Relative expression levels of BnMPK4 and PDF1.2 in Brassica napus cvs. Zhongshuang9 and 84039 were determined by real-time quantitative polymerase chain reaction at 0,6 , and $24 \mathrm{~h}$ after $S$. sclerotiorum inoculation. Values are means of three replicates. Error bars indicate standard deviations. 
pected, PCR results showed two bands originating from two amplified initiation sites in double CaMV 35S (Fig. 3A). Southern blot showed one or two copies inserted in the genome of oilseed rape (Fig. 3B). Northern hybridization results showed that the BnMPK4 RNA level was much higher in all five transgenic plants than the untransformed control (Fig. 3C).
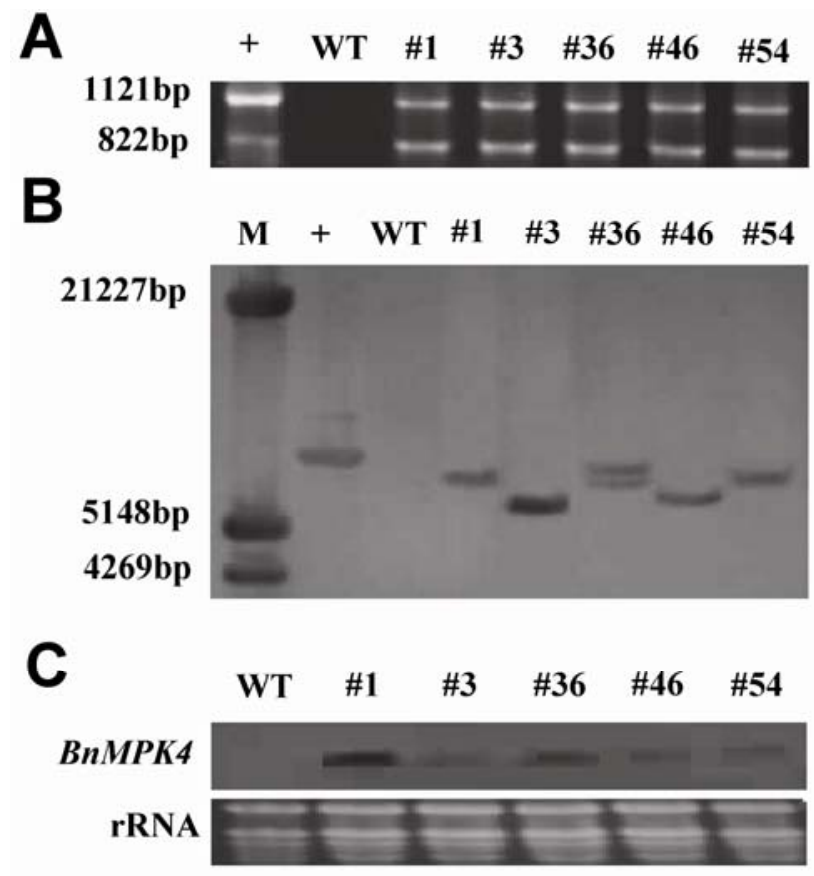

D \#3 WT

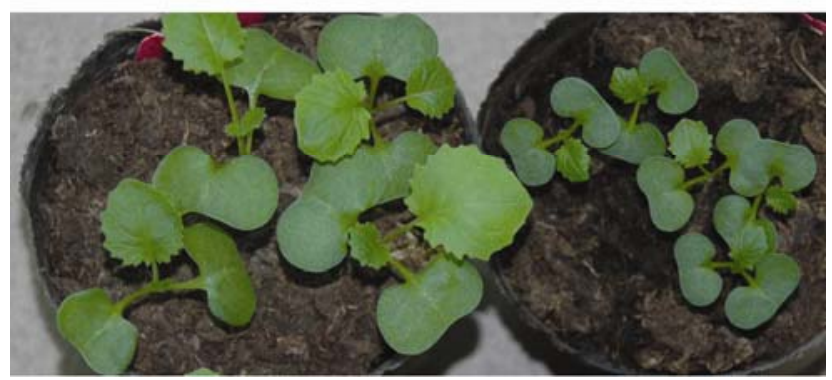

Fig. 3. Transformation of oilseed rape (Brassica napus) with BnMPK4. WT is the untransformed wild-type control; numbers 1, 3, 36, 46, and 54 are transgenic plants. A, Polymerase chain reaction analysis of BnMPK4 transgenic plants with one primer specific to Cauliflower mosaic virus (CaMV) $35 S$ promoter and the other specific to the BnMPK4 cDNA; +, positive control (pG4A-BnMPK4). Because of two amplified initiation sites in double CaMV $35 S$ promoter, two bands are shown. B, Southern blot analysis of BnMPK4 transgenic plants. M, DNA marker; +, the vector pG4A-BnMPK4. C, Northern blot analysis to detect BnMPK4 expression. Ethidium bromide staining of rRNA is used as a loading control. D, $B n M P K 4$ transgenic plants exhibit plant size increase. Seed of BnMPK4 transgenic T1 plants (only no. 3 is shown) and the WT control were sown at the same time in a plant growth room.
Interestingly, we observed that all transgenic lines exhibited an increase in plant size, including larger leaves and higher height, compared with the untransformed line (Fig. 3D).

\section{BnMPK4 transgenic plants show enhanced resistance to $S$. sclerotiorum.}

To test the effect of the transgene on resistance to $S$. sclerotiorum, detached leaves from the five T0 generation transgenic plants and the untransformed control were challenge inoculated with $S$. sclerotiorum mycelia plugs. The results indicated that transgenic plants exhibited delayed disease lesion occurrence and significantly $(P<0.05)$ smaller lesion sizes when compared with the untransformed control (Fig. 4; Supplementary Fig. 2). Soft-rotting necrosis occurred in the untransformed control as early as $24 \mathrm{~h}$ postinoculation but, in transgenic plants, necrosis occurred at 48 or 72 h. At $96 \mathrm{~h}$ postinoculation, lesion sizes of the independent transformants no. $1,3,36,46$, and 54 were reduced by $40.0,40.5,34.6,50.3$, and $50.8 \%$, respectively, compared with those of the untransformed control (Fig. 4).

The enhanced resistance was confirmed in the T1 generation. The T1 plants were selected by spraying herbicide glufosinate and confirmed by PCR for the presence of the BnMPK4 transgene. These PCR-positive plants and their untransformed control at the six-true-leaf stage were used for inoculation with S. sclerotiorum mycelial plugs. From 48 to $120 \mathrm{~h}$ postinoculation, lesion sizes of all the three transgenic lines tested were significantly smaller $(P<0.05)$ than those of the untransformed control (Fig. 5), and these differences became bigger $72 \mathrm{~h}$ postinoculation and afterward. There were no significant $(P<$ $0.05)$ differences between these transgenic lines. In addition, resistance of transgenic line no. 3 tested to $S$. sclerotiorum was significantly higher $(P<0.05)$ than that of resistant cv. Zhongshuang9 (Supplementary Fig. 3). It is highly unlikely that the enhanced resistance of all five independent transgenic plants was simultaneously caused by T-DNA insertion mutations. Therefore, the enhanced resistance should have been caused by $B n M P K 4$ overexpression.

To investigate whether the smaller lesion sizes in transgenic plants originated from lesion delay occurrence or slow expansion rates, lesion development after inoculation was analyzed by constructing a logistic equation of lesion size against time from inoculation (Table 1). The data showed that time until the fastest rate of lesion expansion ( $50 \%$ of the final value) was delayed by 21 to $25 \mathrm{~h}$ in transgenic lines compared with the untransformed control. The values of the slopes, including slope 1 at the point of maximum lesion expansion and slope 2 of the linear regression, indicated that the rate of lesion expansion was slower in transgenic lines than in the untransformed control. These results suggested that enhanced resistance in the transgenic plants is due to both delay of lesion occurrence and slow lesion expansion rate.

The development of S. sclerotiorum mycelia inside leaves of the transgenic plants and the untransformed control was investigated using trypan blue staining. In contrast to the un-
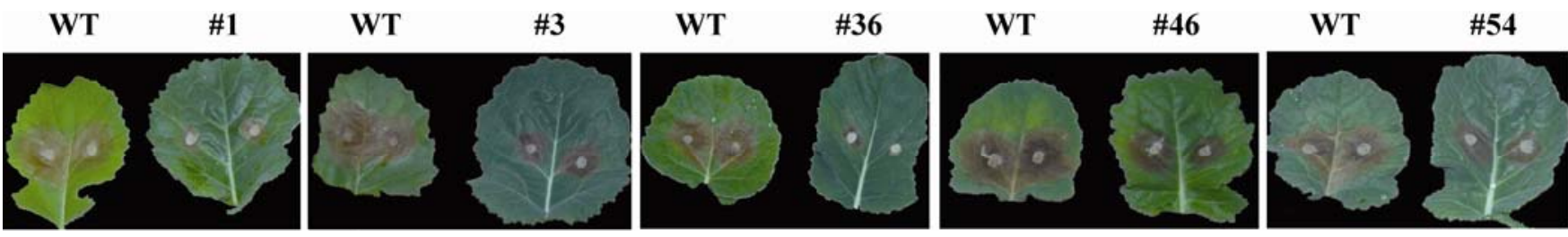

Fig. 4. Resistance of BnMPK4 transgenic T0 plants (Brassica napus) to Sclerotinia sclerotiorum. Transgenic plants no. 1, 3 , 36, 46, and 54 and the untransformed wild type (WT) control were inoculated with the fungal mycelial plugs on detached leaves from six-leaf-old seedlings. Inoculated leaves were arrayed in a container covered with a large polyethylene bag and incubated in a plant growth room at $20 \pm 2{ }^{\circ} \mathrm{C}$. Disease lesion sizes shown were at $96 \mathrm{~h}$ after inoculation. The experiment was repeated twice with similar results. 
transformed control, transgenic plants restricted mycelial growth (Fig. 6). In the untransformed control leaves, mycelial growth was normal and looked like that observed on potato dextrose agar (PDA) whereas, in the transgenic plants, mycelia became curled, shorter, and thicker. These observations, consistent with the above results, clearly indicated that overexpression of BnMPK4 inhibited S. sclerotiorum mycelial growth in oilseed rape.

\section{BnMPK4 transgenic plants show enhanced resistance to Botrytis cinerea.}

Botrytis cinerea causes gray mold diseases in a broad range of plant species, including $B$. napus, and is one of the widely studied necrotrophic plant pathogens. Thus, we tested whether overexpression of BnMPK4 conferring resistance to S. sclerotiorum is also effective against Botrytis cinerea. Inoculation was done on intact plants based on a method described by Audenaert and associates (2002). Results showed that the transgenic plants exhibited higher resistance to Botrytis cinerea than the untransformed control. Fewer spreading lesions and smaller lesions appeared in transgenic plants than in the control (Fig. 7A) and the difference in percent spreading lesions was significant $(P<0.05) 96 \mathrm{~h}$ after inoculation (Fig. 7B). In transgenic lines, 50 to $64 \%$ of lesions were spreading lesions whereas, in the control, $92 \%$ were spreading lesions (i.e. 28 to $42 \%$ reduction in percent spreading lesions in transgenic lines) (Fig. 7B).

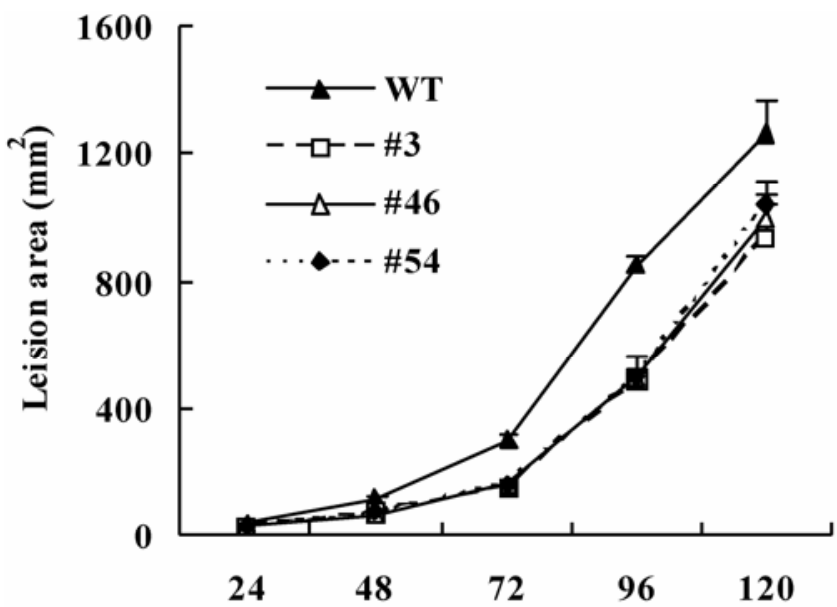

Time after inoculation (hours)

Fig. 5. Resistance of BnMPK4 transgenic T1 plants (Brassica napus) to Sclerotinia sclerotiorum. BnMPK4 transgenic T1 lines no. 3, 46, and 54 and the untransformed wild-type (WT) control were inoculated with the fungal mycelial plugs on leaves of six-leaf-old seedlings. Inoculated leaves were arrayed in a randomized complete block design with three replicates in containers covered with large polyethylene bags and incubated in a plant growth room at $20 \pm 2^{\circ} \mathrm{C}$. Disease progression is shown from 24 to $120 \mathrm{~h}$ after inoculation. Error bars indicate standard deviations.

High levels of $B n M P K 4$ transcription activate $P D F 1.2$ expression but suppress $P R-1$ expression.

To investigate whether $B n M P K 4$ overexpression leads to expression changes in defense-marker genes, we detected transcription abundance of $P D F 1.2$ and $P R-1$ using quantitative RT-PCR. While expression levels of $B n M P K 4$ in the progeny of $B n M P K 4$ transgenic plants were significantly elevated by more than 30 -fold, the levels of PDF1.2 in corresponding lines increased by more than fivefold (Fig. 8). In contrast, $P R-1$ expression was reduced to 40 to $50 \%$ of the untransformed control. These results showed that overexpression of BnMPK4 in B. napus leads to activation of the plant defensin gene $P D F 1.2$ in the absence of induction by chemicals or pathogen treatment. $P D F 1.2$ and $P R-1$ are considered as marker genes for the JA-mediated and SA-mediated defense pathways, respectively (Brodersen et al. 2006; Penninckx et al. 1996; Thomma et al. 1998). Thus, our results suggested that BnMPK4 overexpression might enhance

WT

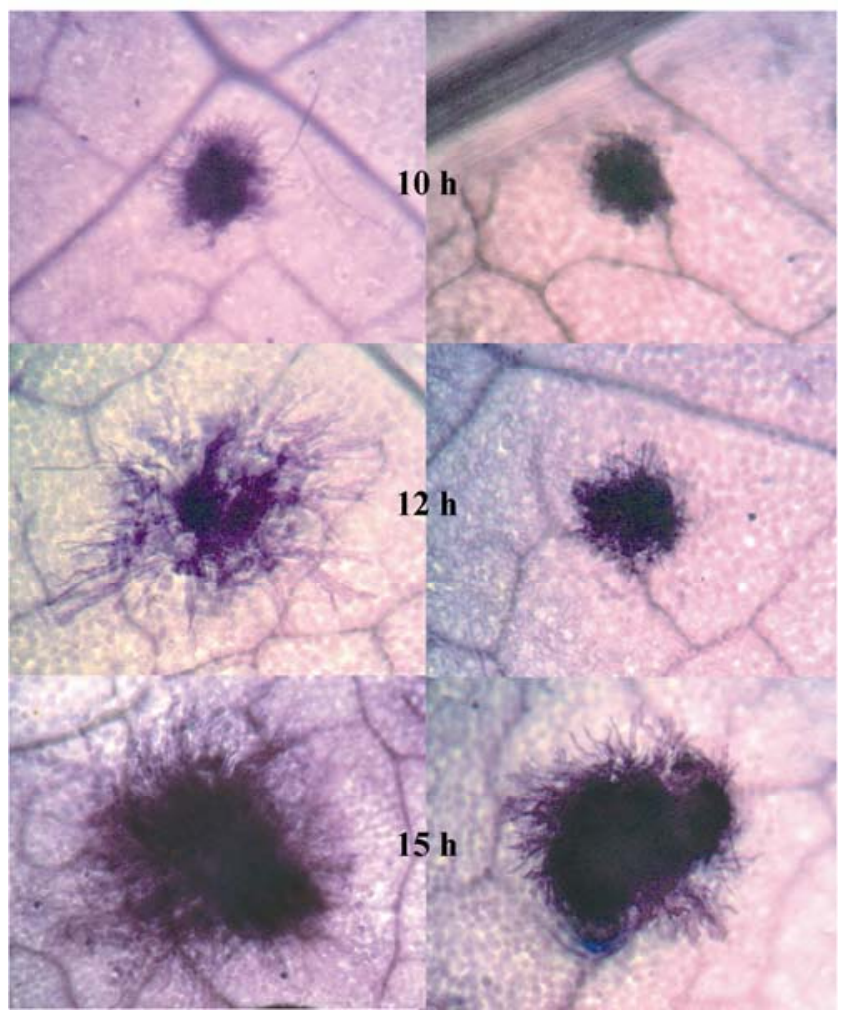

Fig. 6. Inhibition of Sclerotinia sclerotiorum growth by overexpression of BnMPK4 in Brassica napus. Mycelial growth was examined by using trypan blue staining after inoculation on leaves of the BnMPK4 transgenic line no. 3 and the untransformed wild-type (WT) control 10, 12, and $15 \mathrm{~h}$ after inoculation. The experiment was repeated twice with transgenic lines 3,46 , and 54 and results are similar.

Table 1. Logistic analysis of lesion development after inoculation

\begin{tabular}{|c|c|c|c|c|c|}
\hline Line $^{\mathbf{a}}$ & $y=C /\left[1+\exp (-B(t \cdot m)]^{\mathrm{b}}\right.$ & $\mathbf{r}^{\mathbf{c}}$ & $m$ & Slope 1 & Slope 2 \\
\hline WT & $y=1,300 /[1+\exp (-0.0725[t-80.68])]$ & $-0.9376 *$ & 80.68 & 23.57 & -0.0725 \\
\hline 3 & $y=1,300 /[1+\exp (-0.0496[t-105.78])]$ & $-0.8894 *$ & 105.78 & 16.11 & -0.0496 \\
\hline 46 & $y=1,300 /[1+\exp (-0.0522[t-103.20])]$ & $-0.8900 *$ & 103.20 & 16.96 & -0.0522 \\
\hline 54 & $y=1,300 /[1+\exp (-0.0536[t-101.30])]$ & $-0.8783^{*}$ & 101.30 & 17.42 & -0.0536 \\
\hline
\end{tabular}

${ }^{a}$ WT is the untransformed line cv.84039; numbers 3, 46, and 54 are transgenic lines of T1 generation.

${ }^{\mathrm{b}}$ Logistic function of the form $y=C /[1+\exp (-B(t \bullet m)]$ was fitted to the data of lesion size against time and lesion size $(y)$ was regressed on time $(t)$ from inoculation. Parameters: $C\left(1,300 \mathrm{~mm}^{2}\right)$ defined final value of $y ; m$, time (hours) until $50 \%$ of this final value was reached; Slope 1 , slope at time $m$; Slope 2 , the slope of the linear regression of $y^{\prime}\left(y^{\prime}=\ln [(C-y) / y]\right)$.

${ }^{\mathrm{c}}$ Correlation coefficient; * indicates significant at the $5 \%$ level. 
the JA-mediated defense responses but suppress the SA-mediated defense responses.

\section{Activation of $B n M P K 4$ decreases $\mathrm{H}_{2} \mathrm{O}_{2}$ accumulation.}

Ichimura and associates (2006) reported that AtMEKK1 is essential for activation of MPK4, and that loss of MEKK1 resulted in $\mathrm{H}_{2} \mathrm{O}_{2}$ accumulation. $\mathrm{H}_{2} \mathrm{O}_{2}$ is an important class of reactive oxygen species (ROS) which is involved in a broad range of plant physiological processes, including disease defense. Thus, we examined whether BnMPK4 overexpression changes the $\mathrm{H}_{2} \mathrm{O}_{2}$ level in transgenic plants using 3,3-diaminobenzidine (DAB) staining. The BnMPK4 transgenic plants displayed weaker red-brown precipitate in the cotyledons and true leaves than did the untransformed control (Fig. 9A), indicating lower levels of $\mathrm{H}_{2} \mathrm{O}_{2}$ accumulation in this transgenic line. More $\mathrm{H}_{2} \mathrm{O}_{2}$ accumulation was observed in old leaves than in young leaves and in veins and petioles than in the other parts.

To address whether the decrease in $\mathrm{H}_{2} \mathrm{O}_{2}$ level of BnMPK4 transgenic plants is essential to resistance increase, we treated transgenic plants with exogenous $\mathrm{H}_{2} \mathrm{O}_{2}$ prior to inoculation to investigate change in resistance to $S$. sclerotiorum and Botrytis
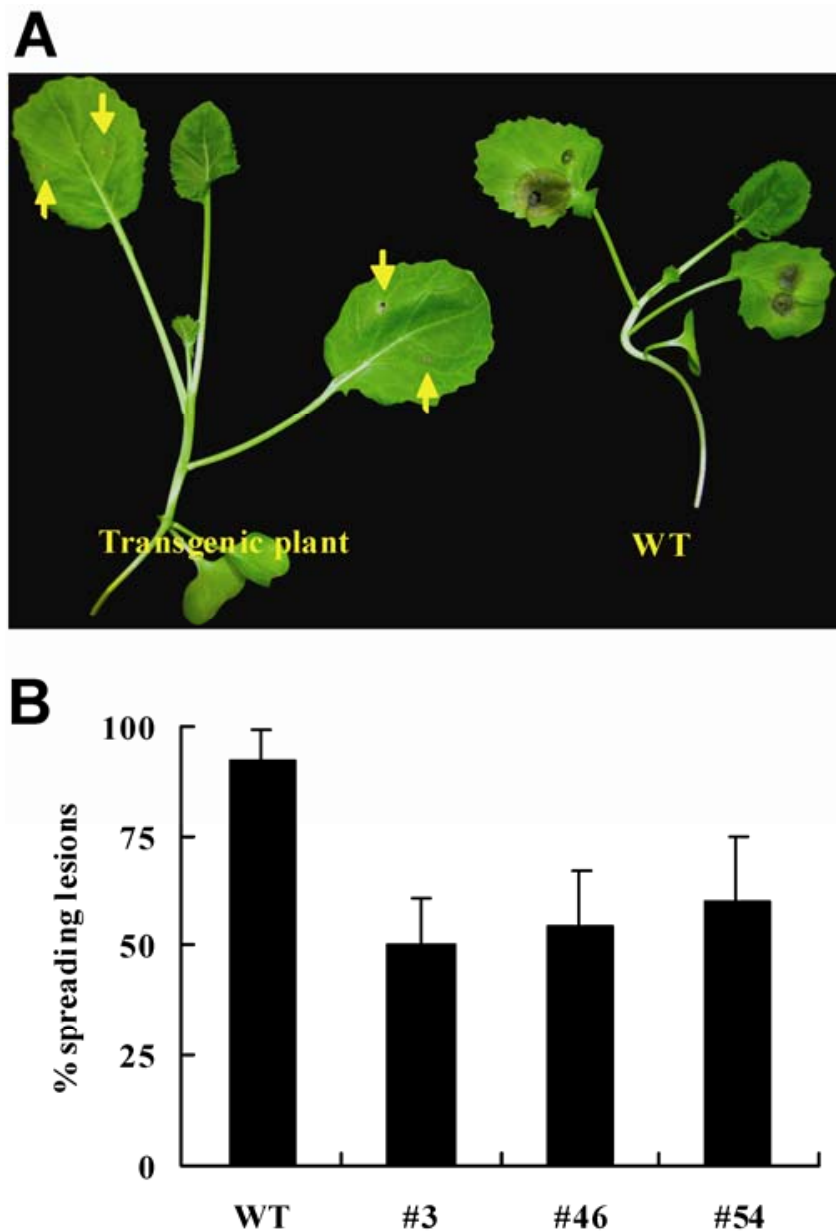

Fig. 7. Resistance of BnMPK4 transgenic plants (Brassica napus) to Botrytis cinerea. BnMPK4 transgenic T1 lines no. 3, 46, and 54 and the untransformed wild-type (WT) control were inoculated with droplets (each 5 $\mu \mathrm{l}$ ) of spore suspension containing $6 \times 10^{5}$ spores $/ \mathrm{ml}, 0.01 \mathrm{M}$ glucose, and $6.7 \mathrm{mM} \mathrm{KH}_{2} \mathrm{PO}_{4}$. Inoculated plants were incubated in a plant growth room at $20 \pm 2{ }^{\circ} \mathrm{C}$ under dark conditions. A, Photo showing four inoculation sites in each plant and difference in disease lesion in a transgenic plant and a WT plant. B, Differences in disease severity in the transgenic lines and a WT control. The experiment was arranged in a randomized complete block design with three replicates. Symptoms were evaluated $96 \mathrm{~h}$ after inoculation and disease severity was expressed as percent spreading lesions for each line. Error bars indicate standard deviations. cinerea. After plant roots were exposed to $\mathrm{H}_{2} \mathrm{O}_{2}$, disease severity caused by both $S$. sclerotiorum and Botrytis cinerea was significantly $(P<0.05)$ greater in $\mathrm{H}_{2} \mathrm{O}_{2}$ treatment than in water treatment (Fig. 9B), indicating enhanced susceptibility when $\mathrm{H}_{2} \mathrm{O}_{2}$ levels increase in these transgenic plants.

\section{DISCUSSION}

Our results provide new data that $B n M P K 4$, a new member of group B, plays a pivotal role in resistance to necrotrophic pathogens $S$. sclerotiorum and Botrytis cinerea, which is most likely mediated by the JA-dependent pathway. Not only is BnMPK4 inducible in a resistant cultivar of $B$. napus after inoculation with $S$. sclerotiorum, but its overexpression in transgenic plants significantly enhances resistance to $S$. sclerotiorum. These new data, which indicate a positive effect on resistance to necrotrophic $S$. sclerotiorum, are complementary to those of the negative regulation role obtained from the MPK4 loss-of-function mutant which exhibits enhanced resistance to the virulent pathogens $P$. syringae and Peronospora parasitica but increased susceptibility to the necrotrophic $A$.
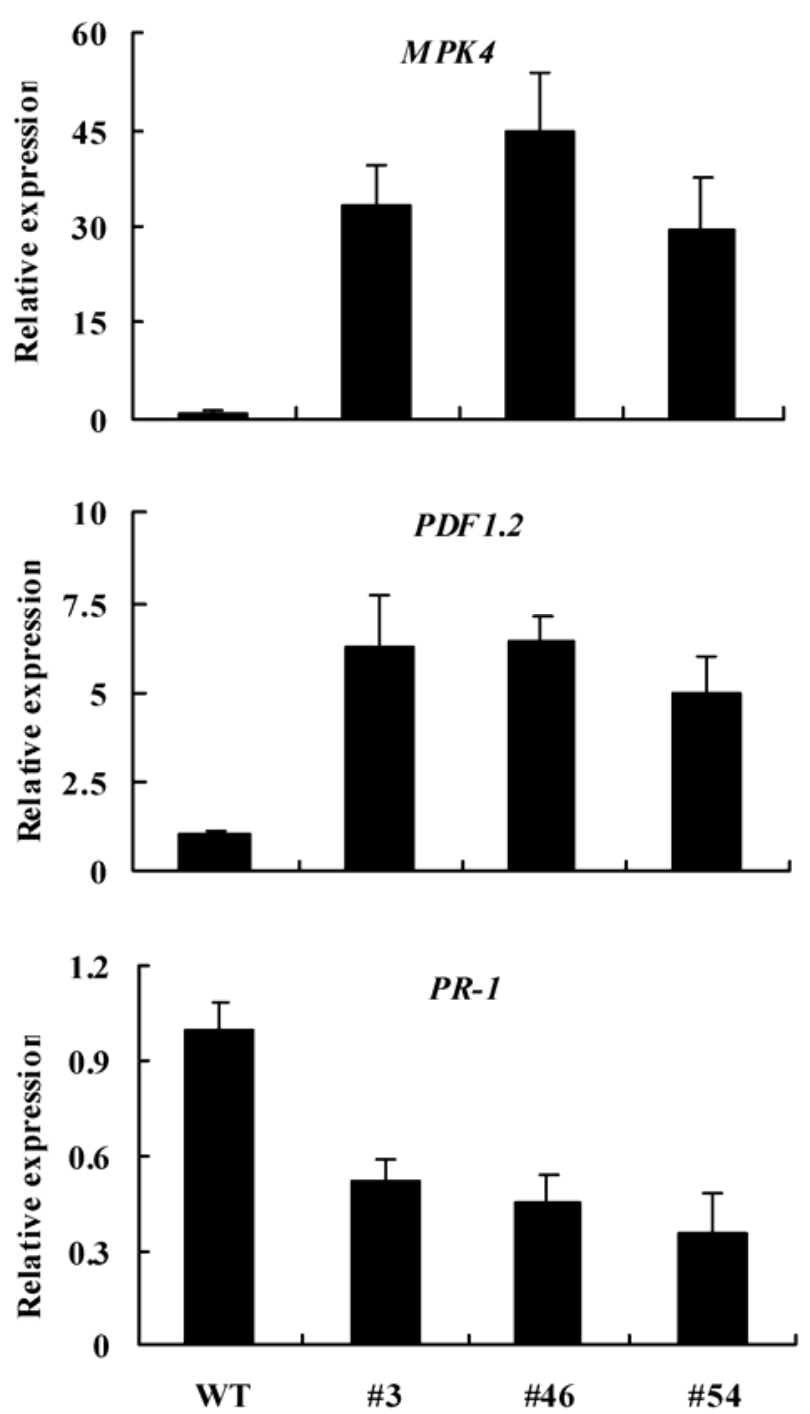

Fig. 8. $P D F 1.2$ activation and $P R-1$ suppression caused by high-level expression of BnMAPK4 in Brassica napus. Relative expression levels of $B n M P K 4, P D F 1.2$, and $P R-1$ in BnMPK4 transgenic plants and the untransformed wild-type (WT) control were quantified by real-time quantitative polymerase chain reaction. Values are means of three replicates. Error bars indicate standard deviations. 
brassicicola (Brodersen et al. 2006; Petersen et al. 2000). Many other MAPK act as a positive regulator of SAR in Arabidopsis, tobacco, and tomato (Asai et al. 2002; del Pozo et al. 2004; Menke et al. 2004; Romeis et al. 1999; Zhang et al. 2007).

Defense-marker gene expression is in an inverse pattern between the Arabidopsis MPK4 mutant and the BnMPK4 transgenic lines. In the Atmpk4 mutant, induction of $P D F 1.2$ is suppressed whereas $P R-1$ and SAR are activated, indicating SA-dependent pathway activation (Brodersen et al. 2006; Petersen et al. 2000). Moreover, in NtMPK4 activity-suppressed tobacco, wound-induced expression of JA-responsive genes is inhibited (Gomi et al. 2005). Interestingly, MEKK1 required for activation of MPK4 also acts as a negative regulator of SAR but inhibits PDF1.2 expression (Ichimura et al. 2006). In contrast, in the $B n M P K 4$ transgenic lines, $P R-1$ transcription is inhibited but expression levels of $P D F 1.2$ are greatly elevated (Fig. 8). These results indicate that MPK4 is required for expression of PDF1.2 in concert with MPK4-activated resistance to necrotrophic pathogens, which supports the idea that MPK4 positively regulates JA-mediated defense responses (Brodersen et al. 2006). It has been suggested that JA/ET-dependent pathway activation can enhance resistance to necrotrophic pathogens, which is associated with expression of PDF1.2 (Feys and Parker 2000; Penninckx et al. 1996; Thomma et al. 1998). For instance, activation of ET responses by ETHYLENE-RESPONSE-FACTOR 1 overexpression in Arabidopsis confers resistance to necrotrophic fungi Botrytis cinerea and Plectosphaerella cucumerina (Berrocal-Lobo et al. 2002); mutations that block JA signaling, including coil (Thomma et al. 1998) and jarl (Ferrari et al. 2003), cause increased susceptibility to the necrotrophic pathogens Botrytis cinerea or A. brassicicola; induced resistance by less aggressive hemibiotrophic fungi Leptosphaeria biglobosa to aggressive hemibiotrophic $L$. maculans is suggested to be activated through the JA-dependent pathway in B. napus (Liu et al. 2007); and results from $S$. sclerotiorum-induced B. napus cDNA microarray (Mao et al. 2007) and Arabidopsis whole-genome microarray (Zhao et al. 2007) suggested that response to $S$. sclerotiorum is mainly JAand ET-dependent in B. napus. Thus, S. sclerotiorum resistance conferred by BnMPK4 might be mediated by the JA-dependent pathway. However, a study based on the Arabidopsis mutant suggested that, in addition to the JA/ET-mediated defense response, the SA-mediated defense response is also involved in defense against $S$. sclerotiorum (Guo and Stotz 2007).

MPK4 might involve redox control to be in favor of resistance enhancement to necrotrophic pathogens. The Atmpk4 mutant, in which $\mathrm{H}_{2} \mathrm{O}_{2}$ levels are strongly elevated (Nakagami et al. 2006), exhibits increased susceptibility to necrotrophic $A$. brassicicola although it constitutively expresses SA-mediated defense responses and exhibits enhanced resistance to the virulent pathogens $P$. syringae and Peronospora parasitica (Brodersen et al. 2006; Petersen et al. 2000). In the present study, $\mathrm{H}_{2} \mathrm{O}_{2}$-level-decreased BnMPK4 transgenic plants exhibited enhanced resistance to necrotrophic S. sclerotiorum and Botrytis cinerea. The previous study showed that the inhibition of $\mathrm{H}_{2} \mathrm{O}_{2}$ accumulation in the host reduces susceptibility to $\mathrm{Bo}$ trytis cinerea (Govrin and Levine 2000). However, the resistance decreased when $\mathrm{H}_{2} \mathrm{O}_{2}$ levels increased by exposing these transgenic plants to $\mathrm{H}_{2} \mathrm{O}_{2}$ solution. This is consistent with the results where spraying various crops with $\mathrm{H}_{2} \mathrm{O}_{2}$ dramatically increased disease severity to Botrytis cinerea (Elad 1992). These are not consistent with the observations in biotrophic pathogen-host interactions that increase of $\mathrm{H}_{2} \mathrm{O}_{2}$ levels as a result of oxidative burst (ROS production) associated with HR or programmed cell death (PCD) can, in general, lead to en-

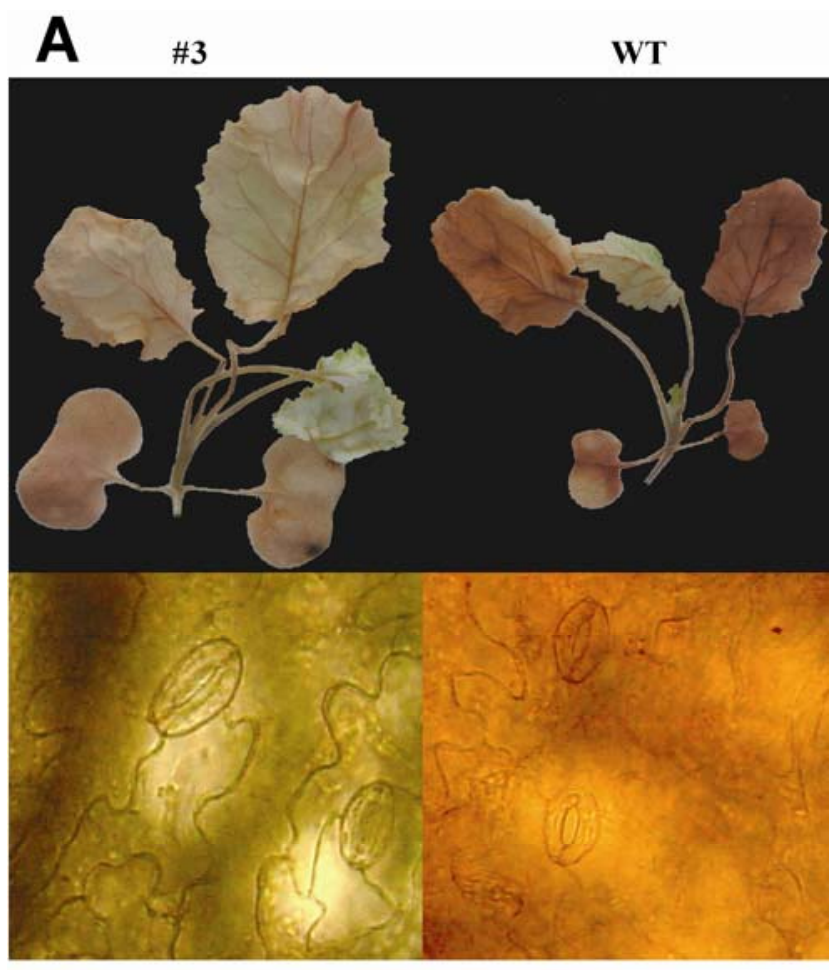

B
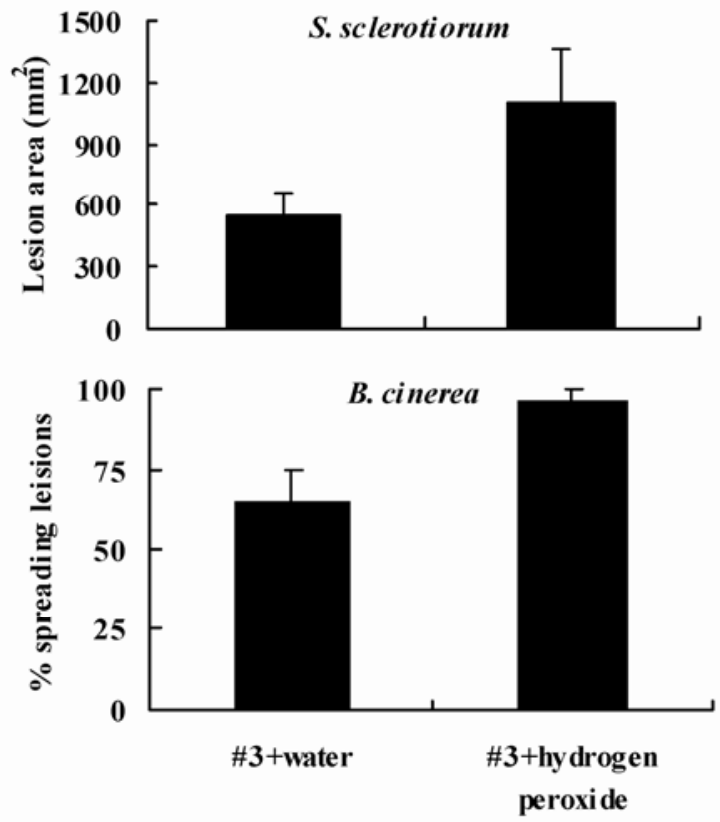

Fig. 9. $\mathrm{H}_{2} \mathrm{O}_{2}$ accumulation in Brassica napus plants overexpressing BnMPK4 and its effect on resistance to Sclerotinia sclerotiorum and Botrytis cinerea. A, Decreases of $\mathrm{H}_{2} \mathrm{O}_{2}$ accumulation in Brassica napus plants overexpressing BnMPK4. In situ detection of $\mathrm{H}_{2} \mathrm{O}_{2}$ was performed by using 3,3-diaminobenzidine staining in the untransformed wild-type (WT) control and the transgenic line no. 3. Lower photos are from closer observation corresponding to upper plants by using a microscope. B, Influence of exogenous $\mathrm{H}_{2} \mathrm{O}_{2}$ on resistance of $B n M P K 4$ transgenic T1 line no. 3 to $S$. sclerotiorum and Botrytis cinerea $96 \mathrm{~h}$ after inoculation. The experiment was arranged in a randomized complete block design with three replicates and symptoms were evaluated $96 \mathrm{~h}$ after inoculation. For $S$. sclerotiorum, plants tested were inoculated with the fungal mycelial plugs on leaves. For Botrytis cinerea, plants tested were inoculated with spore suspension droplets $(5 \mu \mathrm{l})$ containing $6 \times 10^{5}$ spores $/ \mathrm{ml}, 0.01 \mathrm{M}$ glucose, and $6.7 \mathrm{mM} \mathrm{KH}_{2} \mathrm{PO}_{4}$. Disease severity was expressed as percent spreading lesions for each line. Error bars indicate standard deviations. All experiments are repeated in lines 46 and 54 and results are similar. 
hanced host resistance. Furthermore, the cell death driven by ROS seems not to enhance host resistance in necrotrophic pathogen-host interactions. Transgenic tobacco plants expressing negative regulators of mammalian apoptosis lead to markedly enhanced resistance to $S$. sclerotiorum due to cell death inhibition (Dickman et al. 2001). More recently, Kim and associates (2008) reported that, when ROS induction is inhibited, apoptotic-like cell death induced by OA does not occur, and that PCD response is required for disease development. Moreover, the ROS sensitivity of bosl and esal mutants, which showed increased susceptibility to Botrytis cinerea and A. brassicicola, also suggests that tolerance of ROS is important for resistance to necrotrophic fungi (Mengiste et al. 2003). Taking these data together suggests that negative regulation of MPK4 to $\mathrm{H}_{2} \mathrm{O}_{2}$ levels is one way to defend against necrotrophic fungi. On the other hand, MPK4 activation requires $\mathrm{H}_{2} \mathrm{O}_{2}$ (Desikan et al. 2001; Nakagami et al. 2006). The stressinduced activation of MAPK could be explained in most studies by the notion that ROS acts upstream of MAPK pathways. In Arabidopsis, ROS activates expression of MPK3, MPK6, and $M P K 4$, which would sequentially activate plant defense response (Desikan et al. 2001; Kovtun et al. 2000). Thus, there appears to be bidirectional signaling or a loop between MAPK and ROS. Furthermore, elevated levels of $\mathrm{H}_{2} \mathrm{O}_{2}$ via enhanced degradation of $\mathrm{OA}$ in transgenic plants harboring a gene encoding wheat oxalate oxidase increases expression of defenserelated genes (R. Ji, C. Dong, H. Fu, and S. Liu unpublished data) and enhances resistance to $S$. sclerotiorum in B. napus (Dong et al. 2008). Whether these conflicting results imply a concerted control in $\mathrm{H}_{2} \mathrm{O}_{2}$ levels (not to trigger $\mathrm{HR}$ or cell death, but to satisfy requirements for defense activation and normal physiological processes) for resistance in B. napus needs to be studied experimentally.

In summary, this study shows that transgenic oilseed rape overexpressing BnMPK4 significantly enhanced resistance to S. sclerotiorum and Botrytis cinerea, and argues that the kinase might be involved in the employment of different defense pathways. We propose important roles of BnMPK4 and JA/ETmediated defense responses in resistance to $S$. sclerotiorum. The strategies for utilization of BnMPK4 to improve resistance to biotrophic and necrotrophic pathogens are different: the former will be a knock out of the gene and the latter will be overexpression.

\section{MATERIALS AND METHODS}

\section{Plant and fungal materials.}

The B. napus cvs. Zhongshuang9 (highly resistant) and 84039 (highly susceptible to $S$. sclerotiorum) were used in this study. Plants were grown in a plant growth room and the growth conditions were $20 \pm 2{ }^{\circ} \mathrm{C}$ under a photoperiod of 16 and $8 \mathrm{~h}$ at a light intensity of $44 \mu \mathrm{mol} \mathrm{m} \mathrm{m}^{-2} \mathrm{~s}^{-1}$ and 60 to $90 \%$ relative humidity. Botrytis cinerea culture on PDA was provided by G. Li (Huazhong Agricultural University, Wuhan, China) and was isolated from naturally infected oilseed rape leaves. Fresh sclerotia of the fungus $S$. sclerotiorum, collected from oilseed rape stems in the field in Wuhan, China, were germinated to produce hypha inoculum on PDA.

\section{Plasmid construction and plant transformation.}

To construct a vector for constitutive expression of $B n M P K 4$, the vector pG4A was generated by inserting a fragment from pT $24 \mathrm{~A}$ (Wang et al. 2003) containing the CaMV $35 \mathrm{~S}$ enhancer, double CaMV $35 \mathrm{~S}$ promoter, and CaMV Nos terminator into the KpnI-NotI site of pGreen0229 containing a bar gene in its T-DNA region for selecting transgenic plants. A $1.332-\mathrm{kb}$ full-length $B n M P K 4$ cDNA was PCR amplified from its cDNA clone with primers 5'-CGGCTGCAGATTCGTAT CGGCCATTAC-3' and 5'-GCCGCTCGAGAAAACAAAGCA AGACAAA-3', and inserted into the multiple cloning sites (PstI/HindIII) of pG4A, creating a BnMPK4-expressing vector pG4A-BnMPK4. The inserted sequence was confirmed by sequencing. The resulting pG4A-BnMPK4 vector together with the pSoup helper plasmid (Vain et al. 2003) was transformed into the Agrobacterium tumefaciens LBA4404 for plant transformation. B. napus cv. 84039 was grown in a protected field in Wuhan, China and was transformed by in planta Agrobacterium-mediated transformation according to the procedure as described (Xu et al. 2004).

PCR-based screening, Southern and Northern blot analysis.

DNA was extracted from leaves of transformed plants and their offspring. PCR of the MPK4 transgene was carried out with CaMV $35 S$ promoter-specific primer 5'-AGTAGAAAAG GAAGGTGGCA-3' and $M P K 4$-specific primer 5'-AAGGGTT GGTTAGAGCGTAT-3'.

For both Southern and Northern hybridization, the probelabeling, hybridization, and detection were performed with a DIG High Prime DNA Labeling and Detection Starter Kit I (cat. no. 1745832; Roche Applied Science, Mannheim, Germany). Gels were blotted onto Hybond $\mathrm{N}^{+}$membranes (Boehringer Mannheim GmbH, Mannheim, Germany). For Southern blot, genomic DNA was extracted from five independent transgenic T0 plants using an E.Z.N.A plant DNA Mini kit (Omega Bio-tek Inc., Norcross, GA, U.S.A.) and was digested by HindIII for $16 \mathrm{~h}$. Electrophoresis was performed with approximately $20 \mu \mathrm{g}$ of digested genomic DNA for each sample. The hybridization probe was a 485-bp fragment of CaMV $35 \mathrm{~S}$ promoter PCR amplified from pG4A-BnMPK4 using primers 5'-GCCATCATTGCGATAAAGGA-3' and 5'-AAGGATAGTG GGATTGTGCGT-3'. For Northern blot, RNA was isolated from leaves of five independent transgenic T0 plants using the TRIzol reagent (Invitrogen, Carlsbad, CA, U.S.A.). Electrophoresis was performed with approximately $20 \mu \mathrm{g}$ of total RNA in a $1.0 \%$ formaldehyde agarose gel. The hybridization probes were 389-bp fragments generated from PCR amplification of the $B$. napus cDNA using primers 5'-GTATCAGTTGTTGCG TGGGC-3' and 5'-CGTATCTCCTTGCGTTGTCG-3'.

\section{Exogenous $\mathrm{H}_{2} \mathrm{O}_{2}$ application.}

Four-week-old plants were gently washed in water to remove the sand adhering to roots, and the roots were immerged in a solution of $5 \mathrm{mM} \mathrm{H}_{2} \mathrm{O}_{2}$ (Sigma-Aldrich, St. Louis) for $6 \mathrm{~h}$ before inoculation and until experiment end. Treatment with distilled water was used as a control.

\section{Plant inoculation.}

Mycelia of S. sclerotiorum were cultured on PDA. Agar plugs were excised from the edges of growing colonies and upended onto the adaxial surface of plant leaves of six-leaf-old seedlings. For transgenic T0 plants, two leaves detached from each of five transgenic plants were inoculated with two plugs per leaf, each leaf paired with a control leaf of an untransformed plant. For transgenic T1 generation, three independent lines were screened with herbicide Basta $(0.02 \%$ vol/vol) (Bayer, Leverkusen, Germany) by smearing one leaf of each plant. Five days later, these treated leaves were investigated for Basta resistance and leaves of resistant plants were collected for PCR detection. The PCR-positive plants were used for inoculation. Two leaves per plant were inoculated by placing one mycelial plug onto a leaf. The experiment was in a randomized complete block design and repeated three times, with eight plants used in each replicate. Twenty hours after inoculation and at intervals thereafter, lesion sizes were measured in 
length and width and the approximate lesion area (length by width) was calculated for $S$. sclerotiorum inoculation.

Conidia of Botrytis cinerea were obtained from 10-day-old cultures grown on PDA. An inoculation suspension was prepared containing $6 \times 10^{5}$ conidia/ml in $0.01 \mathrm{M}$ glucose and 6.7 $\mathrm{mM} \mathrm{KH}_{2} \mathrm{PO}_{4}$ (pH 5). Two 5- $\mu$ l droplets were used to inoculate each leaf. Inoculated plants in a randomized complete block design with three replicates were incubated at $20 \pm 2{ }^{\circ} \mathrm{C}$ under dark conditions. At least 60 inoculation sites were evaluated for each treatment. Symptoms were evaluated after $96 \mathrm{~h}$. Lesions were classified into two types: spreading lesions showing progressive development and nonspreading lesions which were no more than droplet size. Percentage of spreading lesions was calculated as disease severity for data statistical analysis.

\section{Mycelia staining with trypan blue.}

Growth of $S$. sclerotiorum on the transgenic plants and the untransformed control was examined by trypan blue staining. Inoculated leaves were soaked with ethanol/chloroform (3/1, $\mathrm{vol} / \mathrm{vol}$ ) for $24 \mathrm{~h}$, stained with trypan blue solution for $4 \mathrm{~h}$, and then incubated for $24 \mathrm{~h}$ in chloral hydrate. Mycelia growth was viewed with a fluorescent microscope (LEICA DMRE, Bensheim, Germany).

\section{RT-PCR analysis.}

For RT and quantitative PCR analysis, total RNA of $B$. napus leaves was extracted from the homogenate using the TRIzol Reagent (Invitrogen). RNA samples were first treated with RQ1 DNase (Promega Corp., Madison, WI, U.S.A.). RT reactions were done in a $50-\mu$ l reaction mixture containing 2 $\mu \mathrm{g}$ of RNA, $0.5 \mu \mathrm{g}$ of $(\mathrm{dT})_{20}$ primer, 200 units of reverse transcriptase (M170A; Promega Corp.), and 25 units of RNasin (Promega Corp.) for $1 \mathrm{~h}$ at $42^{\circ} \mathrm{C}$. Quantitative PCR was performed using SYBR Green Realtime PCR Master Mix (TOYOBO, Osaka, Japan) with $0.8 \mu \mathrm{l}$ of each primer $(10 \mu \mathrm{M})$ and $1 \mu \mathrm{l}$ of 1:20 diluted cDNA template in a $20-\mu \mathrm{l}$ reaction mixture. B. napus actin was used as an internal standard. Quantification of the threshold cycle $\left(\mathrm{C}_{\mathrm{T}}\right)$ values in quantitative PCR analysis was achieved by using the $2^{(-\Delta \Delta C(T))}$ method (Livak and Schmittgen 2001). Primers for actin (AF111812) were 5'-CTGGAATTGCTGACCGTATGAG-3' and 5'-ATC TGTTGGAAAGTGCTGAGGG-3'; primers for BnMPK4 (EU581868) were 5'-TTGTATCAGTTGTTGCGTGGG-3' and 5'-TGAAGTCAGTCTCGGATTTGGTC-3'; primers for PDF1.2 (AY884023) were 5'-CATCACCCTTCTCTTCGCT GC-3' and 5'-ATGTCCCACTTGACCTCTCGC-3'; and primers for $P R-1$ (AY623008) were 5'-ATGCCAACGCTCACAAC CA-3' and 5'-CACGGGACCTACGCCTACT-3'. These primer sets were tested by dissociation curve analysis and verified for the absence of nonspecific amplification.

\section{$\mathrm{H}_{2} \mathrm{O}_{2}$ staining with DAB.}

$\mathrm{H}_{2} \mathrm{O}_{2}$ accumulation was visualized by DAB staining. Seedlings were soaked with DAB solution $(1 \mathrm{mg} / \mathrm{ml}, \mathrm{pH} 3.8)$ for 8 $\mathrm{h}$ and then incubated overnight in $96 \%$ (vol/vol) ethanol. DAB was polymerized locally in the presence of $\mathrm{H}_{2} \mathrm{O}_{2}$, giving a visible brown stain.

\section{Statistical analysis.}

Variance analysis was done using the SAS program (SAS Institute Inc., Cary, NC, U.S.A.). For Sclerotinia disease development in T1 transgenic plants, the logistic function of the form $y=C /[1+\exp (-B(t-m)]$ was fitted to the data of lesion size against time, which was done using Genstat ver. 6 (Payne 2000). The lesion sizes (y) were regressed on time after challenge inoculation $(t)$ for each transgenic line and the control.
Parameter $C\left(1,300 \mathrm{~mm}^{2}\right)$ defined final value of $y$ (the lesion size by the end of the experiment); $m$ was time from inoculation to time when $50 \%$ of the final value of $y$ was reached; and $C B / 4$ was slope at time $m$ (the point of inflexion of the logistic curve). Defining $y^{\prime}=\ln (C-y) / y$ then gives the linear regression equation: $y^{\prime}=m B-B t$, where $-B$ was the slope of the linear regression of $y^{\prime}$.

\section{ACKNOWLEDGMENTS}

This work was supported by grants from the Hi-Tech Research and Development Program of China (2006AA10A112), the National Natural Science Foundation of China (30671344), and the Industry Plan (nyhyzx07054) of the Ministry of Agriculture. We thank G. Li for providing Botrytis cinerea isolate and X. Guo, L. Shi, L. Xu, M. Zhao, Z. Wang, X. Zhang, J. Fang, X. Fu, and Y. Liu for their technical assistance.

\section{LITERATURE CITED}

Asai, T., Tena, G., Plotnikova, J., Willmann, M. R., Chiu, W. L., GomezGomez, L., Boller, T., Ausubel, F. M., and Sheen, J. 2002. MAP kinase signalling cascade in Arabidopsis innate immunity. Nature 415:977983.

Audenaert, K., De Meyer., and G. B., and Höfte, M. M. 2002. Abscisic acid determines basal susceptibility of tomato to Botrytis cinerea and suppresses salicylic acid-dependent signaling mechanisms. Plant Physiol. 128:491-501.

Berrocal-Lobo, M., Molina, A., and Solano, R. 2002. Constitutive expression of ETHYLENE-RESPONSE-FACTOR 1 in Arabidopsis confers resistance to several necrotrophic fungi. Plant J. 29:23-32.

Boland, G. J., and Hall, R. 1994. Index of plant hosts of Sclerotinia sclerotiorum. Can. J. Plant Pathol. 16:93-108.

Brader, G., Djamei, A., Teige, M., Palva, T. E., and Hirt, H. 2007. The MAP kinase kinase MKK2 affects disease resistance in Arabidopsis. Mol. Plant-Microbe Interact. 20:589-596.

Brodersen, P., Petersen, M., Nielsen, H. B., Zhu, S., Newman M.-A., Shokat, K. M., Rietz S., Parker J., and Mund J. 2006. Arabidopsis MAP kinase 4 regulates salicylic acid- and jasmonic acid/ethylene-dependent responses via EDS1 and PAD4. Plant J. 47:532-546.

del Pozo, O., Pedley, K. F., and Martin, G. B. 2004. MAPKKK $\alpha$ is a positive regulator of cell death associated with both plant immunity and disease. EMBO (Eur. Mol. Biol. Organ.) J. 23:3072-3082.

Desikan, R., Hancock, J. T., Ichimura, K., Shinozaki, K., and Neill, S. J. 2001. Harpin induces activation of the Arabidopsis mitogen-activated protein kinases AtMPK4 and AtMPK6. Plant Physiol. 126:1579-1587.

Dickman, M. B., Park, Y. K., Oltersdorf, T., Li, W., Clemente, T., and French, R. 2001. Abrogation of disease development in plants expressing animalantiapoptotic genes. Proc. Natl. Acad. Sci. U.S.A. 98:69576962.

Dong, X. 1998. SA, JA, ethylene, and disease resistance in plants. Curr. Opin. Plant Biol. 1:316-323.

Dong, X., Ji R., Guo, X., Foster, S. J., Chen, H., Dong, C., Liu, Y., Hu, Q. and Liu, S. 2008. Expressing a gene encoding wheat oxalate oxidase enhances resistance to Sclerotinia sclerotiorum in oilseed rape. Planta 228:331-340.

Elad, Y. 1992. The use of antioxidants (free radical scavengers) to control grey mould (Botrytis cinerea) and white mould (Sclerotinia sclerotiorum) in various crops. Plant Pathol. 41:417-426.

Felsenstein, J. 1989. Mathematics vs. evolution: Mathematical evolutionary theory. Science 246:941-942.

Ferrari, S., Plotnikova, J. M., De Lorenzo, G., and Ausubel, F. M. 2003. Arabidopsis local resistance to Botrytis cinerea involves salicylic acid and camalexin and requires EDS4 and PAD2, but not SID2, EDS5 or PAD4. Plant J. 35:193-205.

Feys, B. J., and Parker, J. E. 2000. Interplay of signaling pathways in plant disease resistance. Trends Genet. 16:449-455.

Frye, C. A., Tang, D. D., and Innes, R. W. 2001. Negative regulation of defense responses in plants by a conserved MAPKK kinase. Proc. Natl. Acad. Sci. U.S.A. 98:373-378.

Glazebrook J. 2005. Contrasting mechanisms of defense against biotrophic and necrotrophic pathogens. Annu. Rev. Phytopathol. 43:205-227.

Glazebrook, J., Chen, W., Estes, B., Chang, H.-S., Nawrath, C., Métraux, J.-P., Zhu, T., and Katagiri, F. 2003. Topology of the network integrating salicylate and jasmonate signal transduction derived from global expression phenotyping. Plant J. 34:217-228.

Gomi, K., Ogawa, D., Katou, S., Kamada, H., Nakajima, N., Saji, H., Soyano, T., Sasabe, M., Machida, Y., Mitsuhara, I., Ohashi, Y., and Seo, 
S. 2005. A mitogen-activated protein kinase NtMPK4 activated by SIPKK is required for jasmonic acid signaling and involved in ozone tolerance via stomatal movement in tobacco. Plant Cell Physiol. 46:1902-1914.

Govrin, E. M., and Levine, A. 2000. The hypersensitive response facilitates plant infection by the necrotrophic pathogen Botrytis cinerea. Curr. Biol. 10:751-757.

Grant J. J., Yun, B., and Loake, G. J. 2000. Oxidative burst and cognate redox signaling reported by luciferase imaging: Identification of a signal network that functions independently of ethylene, SA and MeJA but is dependent on MAPKK activity. Plant J. 24:569-582.

Guo, X., and Stotz, H. U. 2007. Defense against Sclerotinia sclerotiorum in Arabidopsis is dependent on jasmonic acid, salicylic acid, and ethylene signaling. Mol. Plant-Microbe Interact. 20:1384-1395.

Hammerschmidt, R. 1999. Induced disease resistance: How do induced plants stop pathogens? Physiol. Mol. Plant Pathol. 55:77-84.

Ichimura, K., Tena, G., Henry, Y., Zhang, Z., Hirt, H., Wilson, C., Morris, P., Mundy, J., Innes, R., Ecker, J., Scheel, D., Klessig, D. F., Machida, Y., Mundy, J., Ohashi, J., and Walker, J. C. 2002. Mitogen-activated protein kinase cascades in plants: A new nomenclature. Trends Plant Sci. 7:301-308.

Ichimura, K., Casais C., Peck, S. C., Shinozaki, K., and Shirasu, K. 2006. MEKK1 is required for MPK4 activation and regulates tissue-specific and temperature-dependent cell death in Arabidopsis. J. Biol. Chem. 281:36969-36976.

Kim, K. S., Min, J.-Y., and Dickman, M. B. 2008. Oxalic acid is an elicitor of plant programmed cell death during Sclerotinia sclerotiorum disease development. Mol. Plant-Microbe Interact. 21:605-612.

Kovtun, Y., Chiu, W. L., Tena, G., and Sheen, J. 2000. Functional analysis of oxidative stress-activated mitogen-activated protein kinase cascade in plants. Proc. Natl. Acad. Sci. U.S.A. 97:2940-2945.

Liu, S., Wang, H., Zhang, J., Fitt, B. D., Xu, Z., Evans, N., Liu, Y., Yang, W., and Guo, X. 2005. In vitro mutation and selection of doubled-haploid Brassica napus lines with improved resistance to Sclerotinia sclerotiorum. Plant Cell Rep. 24:133-144.

Liu, S., Liu, R., Latunde-Dada, A. O., Cools, H. J., Foster, S. J., Huang, Y J., and Fitt, B. D. L. 2007. Comparison of Leptosphaeria biglobosainduced and chemically induced systemic resistance to L. maculans in Brassica napus. Chin. Sci. Bull. 52:1053-1062.

Livak, K. J., and Schmittgen, T. D. 2001. Analysis of relative gene expression data using real-time quantitative PCR and the $2^{(-\Delta \Delta C(T))}$ method. Methods 25:402-408.

Madhani, H. D., Styles, C. A., and Fink, G. R. 1997. MAP kinases with distinct inhibitory functions impart signaling specificity during yeast differentiation. Cell 91:673-684.

Mao, H., Dong, C., Su Z., Ji, R., Guo, X., and Liu, S. 2007. Analysis of a set of 14,549 Brassica napus unique ESTs and signature genes related to Sclerotinia resistance. Pages 138-140 in: Proc. 12th Int. Rapeseed Congr. Vol. II, Biotechnology. Science Press USA Inc., Monmouth Junction, NJ, U.S.A.

Martinez, C., Blanc, F., Le Claire, E., Besnard, O., Nicole, M., and Baccou, J.-C. 2001. Salicylic acid and ethylene pathways are differentially activated in melon cotyledons by active or heat-denatured cellulase from Trichoderma longibrachiatum. Plant Physiol. 127:334-344.

Mengiste, T., Chen, X., Salmeron, J., and Dietrich, R. 2003. The BOTRYTIS SUSCEPTIBLE1 gene encodes an R2R3MYB transcription factor protein that is required for biotic and abiotic stress responses in Arabidopsis. Plant Cell 15:2551-2565.

Menke, L. H., van, Pelt, J. A., Pieterse, C. M. J., and Klessig, D. F. 2004. Silencing of mitrogen-activated protein kinase MPK6 comprises disease resistance in Arabidopsis. Plant Cell 16:897-907.
Nakagami, H., Soukupová, H., Schikora, A. Zársk, V., and Hirt H. 2006. A mitogen-activated protein kinase kinase kinase mediates reactive oxygen species homeostasis in Arabidopsis. J. Biol. Chem. 281:38697-38704.

Payne, R. W., 2000. The Guide to GENSTAT: Part 2. Oxford University Press, Oxford.

Pedley, K. F., and Martin, G. B. 2005. Role of mitogen-activated protein kinases in plant immunity. Curr. Opin. Plant Biol. 8:541-547.

Penninckx, I. A., Eggermont, K., Terras, F. R., Thomma, B. P., De Samblanx, G. W., Buchala, A., Métraux, J. P., Manners, J. M., and Broekaert, W. F. 1996. Pathogen-induced systemic activation of a plant defensin gene in Arabidopsis follows a salicylic acid-independent pathway. Plant Cell 8:2309-2323.

Petersen, M., Brodersen, P., Naested, H. Andreasson E., Lindhart, U., Johansen, B., Nielsen, H. B., Lacy, M., Austin, M. J., Parker, J. E., Sharma, S. B., Klessig, D. F., Martienssen, R. Mattsson, O., Jensen, A. B., and Mundy, J. 2000. Arabidopsis MAP kinase 4 negatively regulates systemic acquired resistance. Cell 103:1111-1120.

Ren, D., Liu, Y., Yang, K. Y., Han, L., Mao, G., Glazebrook, J., and Zhang, S. 2008. A fungal-responsive MAPK cascade regulates phytoalexin biosynthesis in Arabidopsis. Proc. Natl. Acad. Sci. U.S.A. 105:5638-5643.

Romeis, T., Piedras, P., Zhang, S., Klessig, D. F., Hirt, H., and Jones, J. D. 1999. Rapid Avr9- and Cf-9-dependent activation of MAP kinases in tobacco cell cultures and leaves: Convergence of resistance gene, elicitor, wound, and salicylate responses. Plant Cell 11:273-287.

Takemoto, D., Hardham, A. R., and Jones, D. A. 2005. Differences in cell death induction by Phytophthora Elicitins are determined by signal components downstream of MAP kinase kinase in different species of Nicotiana and cultivars of Brassica rapa and Raphanus sativus. Plant Physiol. 138:1491-504.

Thomma, B. P., Eggermont, K., Penninckx, I. A., Mauch-Mani, B., Vogelsang, R., Cammue, B. P., and Broekaert, W. F. 1998. Separate jasmonate-dependent and salicylate-dependent defense-response pathways in Arabidopsis are essential for resistance to distinct microbial pathogens. Proc. Natl. Acad. Sci. U.S.A. 95:15107-11.

Vain, P., Afolabi, A. S., Worland, B. and Snape J. W. 2003. Transgene behaviour in populations of rice plants transformed using a new dual binary vector system: pGreen/pSoup. Theor. Appl. Genet. 107:210-217.

Wang, Z. X., Liu, Y. H., Sun, J. S., and Jia, S. R. 2003. Cloning of glucose oxidase gene and expression in tobacco plants. Progr. Nat. Sci. 13:248252.

Wu, J., Hettenhausen, C., Meldau, S., and Baldwin, I. T. 2007. Herbivory rapidly activates MAPK signaling in attacked and unattacked leaf regions but not between leaves of Nicotiana attenuata. Plant Cell 19:1096-122.

Xu, G.-S., Rao, Y.-Q., Chen, Y., Zhang, C.-Y., and Meng J.-L. 2004. Genetic transformation of Brassica napus with in planta method. Acta. Agron. Sin. 30:1-5.

Zhang, X., Dai, Y., Xiong, Y., DeFraia, C., Li, J., Dong, X., and Mou, Z. 2007. Overexpression of Arabidopsis MAP kinase kinase 7 leads to activation of plant basal and systemic acquired resistance. Plant J. 52:1066-1079.

Zhao, J., Wang, J., An L., Doerge, R. W., Chen Z. J., Grau C. R. Meng, J., and Osborn, T. C. 2007. Analysis of gene expression profiles in response to Sclerotinia sclerotiorum in Brassica napus. Planta 227:13-24.

\section{AUTHOR-RECOMMENDED INTERNET RESOURCE}

Hypothesis Creator IPSORT Prediction home page: hypothesiscreator.net/iPSORT/\#predict 